\title{
An Optimal Process Control in the Rectification Facilities
}

\author{
Nikolay D. Demidenko and Lyudmila V. Kulagina*b \\ ${ }^{a}$ Nauka Specialized Design Technology Office \\ Institute of Computational Technologies SB RAS \\ 425 office, 53 Mira, Krasnoyarsk, 660049, Russia \\ ${ }^{b}$ Siberian Federal University \\ 79 Svobodny, Krasnoyarsk, 660041, Russia
}

Received 21.12.2016, received in revised form 07.01.2017, accepted 30.01.2017

Presents mathematical models for the process technological furnace and the rectification column. Formulated the the optimal control problem. We obtain necessary optimality conditions. Presents the results of numerical experiments for industrial facilities.

Keywords: mathematical models, process furnaces, rectifying equipment.

Citation: Demidenko N.D., Kulagina L.V. An optimal process control in the rectification facilities, J. Sib. Fed. Univ. Eng. technol., 2017, 10(1), 95-105. DOI: 10.17516/1999-494X-2017-10-1-95-105.

\section{Оптимальное управление технологическим процессом в ректификационных установках}

\author{
Н.Д. Демиденко ${ }^{a}$, Л.В. Кулагина ${ }^{6}$ \\ ${ }^{a}$ Красноярский филиал-СКТБ «Наука» \\ Института вычислительных технологий СО РАН \\ Россия, 660049, Красноярск, Мира, 53 \\ ${ }^{6}$ Сибирский федеральный университет \\ Россия, 660041, Красноярск, Свободный, 79
}

Предложены математические модели для технологической печи и ректификачионной колонны. Сформулированы задачи оптимального управления. Получены необходимые условия оптимальности. Приведены результаты численных экспериментов для промышленных объектов.

Ключевые слова: математические модели, технологические печи, ректификационные установки.

(C) Siberian Federal University. All rights reserved

* Corresponding author E-mail address: klvation@gmail.com 
На нефтеперерабатывающих заводах ведется переработка нефти в бензин, керосин, мазут, смазочные масла, сырье для нефтехимии и т.д. Переработка осуществляется в технологических установках: для первичной переработки, каталитического риформинга, каталитического крекинга и т.д. Основными аппаратами установок являются трубчатые печи, ректификационные колонны и др. Основу работы этих аппаратов составляют процессы теплообмена, массообмена и гидродинамики взаимодействующих потоков [1]. Анализ процессов и проектирование эффективных режимов рассматриваемых объектов химической технологии с целью создания автоматизированных систем контроля и управления является важнейшей проблемой современного производства.

Математические методы и вычислительные средства позволяют осуществить процесс моделирования и оптимизации сложных технологических установок, включающих технологические печи, ректификационные колонны и др. [2-4]. Для описания таких процессов всей установки возможна математическая модель всей цепочки. На рис. 1 приведена принципиальная схема подобной установки. Уже накоплен достаточный опыт исследования отдельных аппаратов и возможен подход к исследованию установки в целом. В практике исследуемые объекты химической технологии рассматриваются как объекты с распределенными параметрами, для описания которых применяется математический аппарат дифференциальных уравнений в частных производных [5]. Для анализа статических и динамических режимов и решения задач оптимального управления формулируются соответствующие краевые задачи.

Ректификационная установка состоит из технологической печи и ректификационных колонн. Трубчатые печи разных конструкций широко распространены в нефтегазоперерабатывающей, нефтехимической и других отраслях промышленности, являются составной частью многих установок и применяются в различных технологических процессах (перегонка нефти и мазута, пиролиз, каталитический крекинг, очистка масел и др.). В печи сырье нагревается до требуемой температуры и подается в среднюю часть колонны для разделения смеси на компоненты.

\section{Постановка задачи для технологических печей}

Если учитывать, что сырье выводится из средней части печи, то, исходя из законов механики сплошных сред, можно получить следующие уравнения нестационарного горения [6-8]:

$$
\left\{\begin{array}{l}
\frac{\partial \rho}{\partial t}=-u \frac{\partial \rho}{\partial l}-\rho \frac{\partial u}{\partial l}, \\
\frac{\partial x}{\partial t}=-u \frac{\partial x}{\partial l}-\frac{x}{\tau}, \\
\frac{\partial u}{\partial t}=-u \frac{\partial u}{\partial l}-R \frac{\partial T_{\mathrm{m}}}{\partial l}-\frac{R T_{\mathrm{n}}}{\rho} \frac{\partial \rho}{\partial l}, \\
\frac{\partial T_{\mathrm{\Pi}}}{\partial t}=(1-\gamma) T_{\mathrm{n}} \frac{\partial u}{\partial l}-u \frac{\partial T_{\mathrm{n}}}{\partial l}+\frac{x q}{C_{\nu} \tau}-\frac{Q\left(T_{\mathrm{n}}\right)}{C_{\nu} \rho}+K_{1}\left(T_{\mathrm{c}}^{1}-T_{\mathrm{n}}\right)+K_{1}\left(T_{\mathrm{c}}^{2}-T_{\mathrm{n}}\right), \\
\frac{\partial T_{\mathrm{c}}^{1}}{\partial t}=\omega \frac{\partial T_{\mathrm{c}}^{1}}{\partial l}+K_{2}\left(T_{\mathrm{n}}-T_{\mathrm{c}}^{1}\right)-Q\left(T_{\mathrm{n}}, T_{\mathrm{c}}^{1}\right), \\
\frac{\partial T_{\mathrm{c}}^{2}}{\partial t}=-\omega \frac{\partial T_{\mathrm{c}}^{2}}{\partial l}+K_{2}\left(T_{\mathrm{n}}-T_{\mathrm{c}}^{2}\right)-Q\left(T_{\mathrm{n}}, T_{\mathrm{c}}^{2}\right) . \\
\quad-96-
\end{array}\right.
$$




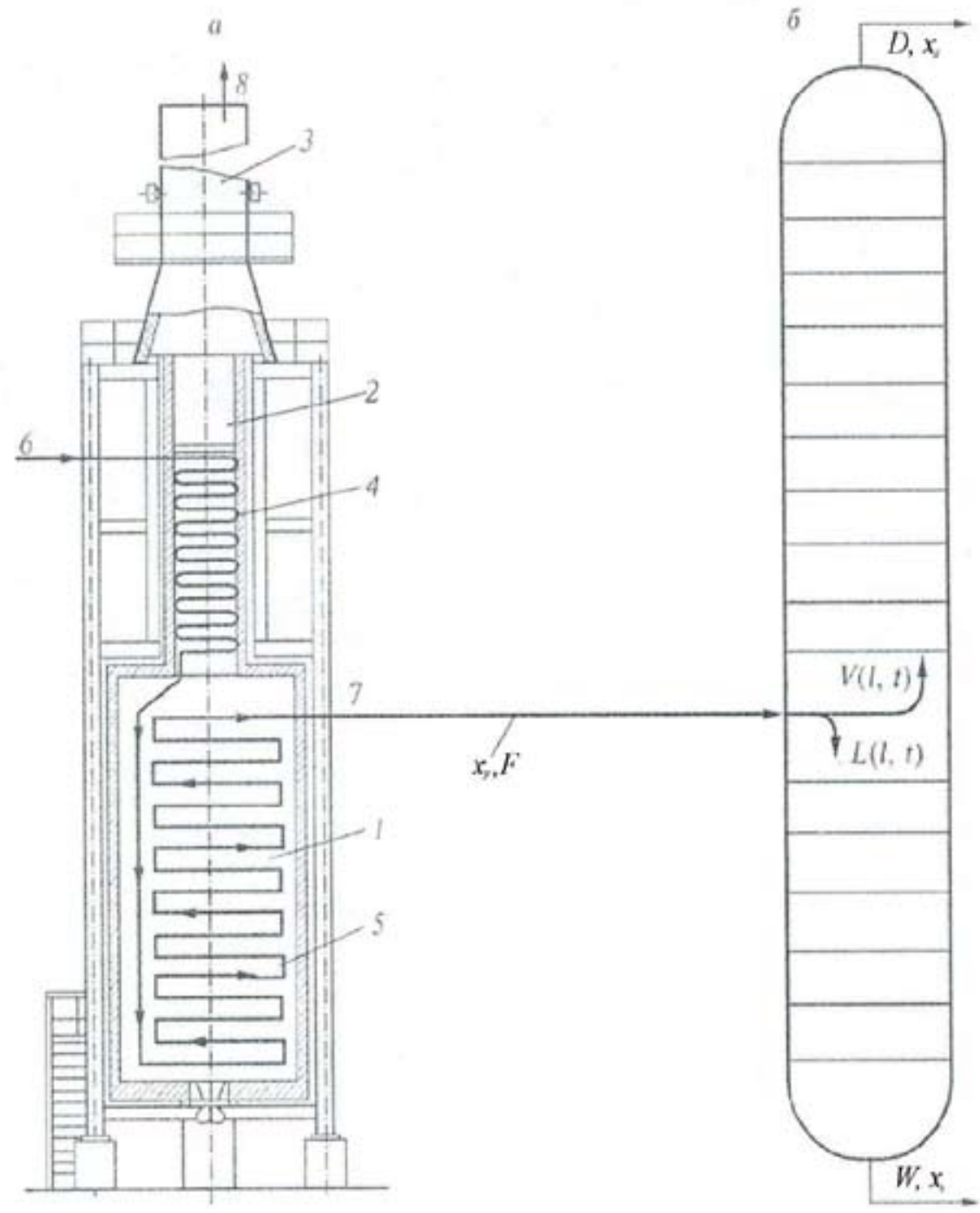

Рис. 1. Схема ректификационной установки: а - технологическая печь, б - ректификационная колонна; 1 - камера радиации (топочная камера); 2 - камера конвекции; 3 - дымовая труба; 4 - конвекционные трубы; 5 - радиантные трубы; 6 - вход сырья; 7 - выход сырья; 8 - дымовые газы

Здесь $x(l, t)$ - концентрация горючего вещества, $\rho(l, t), u(l, t), T_{n}(l, t)$ - плотность, скорость и температура дымовых газов; $T_{c}^{l}(l, t), T_{c}^{2}(l, t)$ - температура нисходящего и восходящего потоков нагреваемого сырья; $t, l$ - временная и пространственная координаты.

Начальные условия

$$
\rho(l, 0)=\rho_{0}, x(l, 0)=x_{0}, u(l, 0)=u_{0}, T_{\mathrm{n}}(l, 0)=T_{\mathrm{n} 0}, T_{\mathrm{c}}^{1}(l, 0)=T_{\mathrm{c} 0}^{1}, T_{\mathrm{c}}^{2}(l, 0)=T_{\mathrm{c} 0}^{2} .
$$

Граничные условия

$$
\begin{aligned}
& \rho(0, t)=\varphi_{1}(t), x(0, t)=\varphi_{2}(t), u(0, t)=\varphi_{3}(t), T_{\mathrm{n}}(0, t)=\varphi_{4}(t), \\
& T_{\mathrm{c}}^{1}(L, t)=\varphi_{5}(t), T_{\mathrm{c}}^{2}(0, t)=T_{\mathrm{c}}^{1}(0, t) .
\end{aligned}
$$

Здесь $L$ - длина печи. 
Как и в случае оптимизации процессов разделения, здесь могут быть проанализированы различные задачи оптимального управления процессом технологических печей. Рассмотрим одну такую задачу $[1,3]$.

Пусть управляемый процесс технологической печи описывается следующей системой (1).

В качестве управления возьмем изменение плотности горючего $v_{1}$, концентрации $v_{2}$, скорости $v_{3}$, температуры факела $v_{4}$ и сырья $v_{5}$. На управления наложим следующие ограничения:

$$
v_{i \min } \leq v_{i} \leq v_{i \max } .
$$

Введя фиктивные управления $z_{i}, i=1,5$, сведем неравенства (4) к равенствам

$$
\left(v_{i \min }-v_{i}\right)\left(v_{i}-v_{i \max }\right)-z_{i}^{2}=0, i=\overline{1,5} .
$$

Связь граничных условий с управлениями представлена ниже:

$$
\begin{aligned}
& \frac{\partial \rho(0, t)}{\partial t}=b_{1} v_{1}(t), \\
& \frac{\partial x(0, t)}{\partial t}=b_{2} v_{2}(t), \\
& \frac{\partial u(0, t)}{\partial t}=b_{3} v_{3}(t), \\
& \frac{\partial T_{n}(0, t)}{\partial t}=b_{4} v_{4}(t), \\
& \frac{\partial T_{C}^{1}(L, t)}{\partial t}=b_{5} v_{5}(t) .
\end{aligned}
$$

Задача оптимального управления формулируется следующим образом. Найти такие $v_{i}(t)$, $i=\overline{1,5}$, из промежутков (4), которые в силу систем (1)-(3) доставляют минимум критерия качества:

$$
\int_{0}^{T} \int_{0}^{L}\left(T_{C}^{1}(l, t)-T_{C}^{1^{*}}(l, t)\right)^{2} \mathrm{~d} l \mathrm{~d} t .
$$

\section{Необходимые условия оптимальности}

Введем обозначения:

$$
\begin{aligned}
& \frac{\partial \rho}{\partial l}=\varsigma^{(1)}, \frac{\partial x}{\partial l}=\varsigma^{(2)}, \\
& \frac{\partial u}{\partial l}=\varsigma^{(3)}, \\
& \frac{\partial T_{\Pi}}{\partial l}=\varsigma^{(4)}, \frac{\partial T_{C}^{1}}{\partial l}=\varsigma^{(5)}, \frac{\partial T_{C}^{1}}{\partial l}=\varsigma^{(5)}, \quad \frac{\partial T_{C}^{2}}{\partial l}=\varsigma^{(6)} .
\end{aligned}
$$

С учетом (7) система (1) будет иметь вид 


$$
\left\{\begin{array}{l}
\frac{\partial \rho}{\partial t}=-u \varsigma^{(1)}-\rho \varsigma^{(3)} \equiv X_{1}, \\
\frac{\partial x}{\partial t}=-u \varsigma^{(2)}-\frac{x}{\tau} \equiv X_{2}, \\
\frac{\partial u}{\partial t}=-u \varsigma^{(3)}-R \varsigma^{(4)}-\frac{R T}{\rho} \varsigma^{(1)} \equiv X_{3}, \\
\frac{\partial T_{\Pi}}{\partial t}=(1-\gamma) T_{\text {п }} \varsigma^{(3)}=\frac{x q}{C_{\nu} \tau}-\frac{Q\left(T_{\pi}, T_{C}^{1}, T_{C}^{2}\right)}{C_{\nu} \rho}-u \varsigma^{(4)}+K_{1}\left(T_{C}^{1}+T_{C}^{2}-T_{\mathrm{n}}\right) \equiv X_{4}, \\
\frac{\partial T_{C}^{1}}{\partial t}=-\omega \varsigma^{(5)}+K_{2}\left(T-T_{C}^{1}\right)-Q\left(T_{\pi}, T_{C}^{1}\right) \equiv X_{5}, \\
\frac{\partial T_{C}^{2}}{\partial t}=\omega \varsigma^{(6)}+K_{2}\left(T-T_{C}^{2}\right)-Q^{\prime}\left(T_{\pi}, T_{C}^{2}\right) .
\end{array}\right.
$$

Для получения необходимых условий оптимальности рассмотрим вспомогательный функционал

$$
I=I_{1}+I_{2}=\iint_{\Omega} \tilde{L} \mathrm{~d} l \mathrm{~d} t+\int_{\partial \Omega} \tilde{l} \mathrm{~d} t
$$

где

$$
\begin{aligned}
& \tilde{L}=\xi_{1}\left(\frac{\partial \rho}{\partial t}-X_{1}\right)+\xi_{2}\left(\frac{\partial x}{\partial t}-X_{2}\right)+\xi_{3}\left(\frac{\partial u}{\partial t}-X_{3}\right)+ \\
& +\xi_{4}\left(\frac{\partial T_{\mathrm{\Pi}}}{\partial t}-X_{4}\right)+\xi_{5}\left(\frac{\partial T_{C}}{\partial t}-X_{5}\right)+\eta_{1}\left(\frac{\partial \rho}{\partial l}-\xi^{(1)}\right)+ \\
& +\eta_{2}\left(\frac{\partial x}{\partial l}-\xi^{(2)}\right)+\eta_{3}\left(\frac{\partial u}{\partial l}-\xi^{(3)}\right)+\eta_{4}\left(\frac{\partial T_{\mathrm{\Pi}}}{\partial l}-\xi^{(4)}\right)+ \\
& +\eta_{5}\left(\frac{\partial T_{C}}{\partial l}-\xi^{(5)}\right)+\eta_{6}\left(\frac{\partial T_{C}^{2}}{\partial l}-\xi^{(6)}\right)+\left(T_{C}^{1}-T_{C}^{1^{*}}\right)^{2}, \\
& \tilde{l}=\lambda^{(1)}\left[\frac{\partial \rho(0, t)}{\partial t}-b_{1} v_{1}(t)\right]+\lambda^{(2)}\left[\frac{\partial x(0, t)}{\partial t}-b_{2} v_{2}(t)\right]+ \\
& +\lambda^{(3)}\left[\frac{\partial u(0, t)}{\partial t}-b_{3} v_{3}(t)\right]+\lambda^{(4)}\left[\frac{\partial T_{\mathrm{n}}(0, t)}{\partial t}-b_{4} v_{4}(t)\right]+ \\
& +\lambda^{(5)}\left[\frac{\partial T_{C}(0, t)}{\partial t}-b_{5} v_{5}(t)\right]+\lambda^{(6)}\left[T_{C}^{2}(0, t)-T_{C}^{1}(0, t)\right]+ \\
& \quad+\sum_{i=1}^{5} \mu^{1 v}\left[\left(v_{i \max }-v_{i}(t)\right) \cdot\left(v_{i}(t)-v_{\text {imax }}\right)-z_{i}^{2}(t)\right]
\end{aligned}
$$

$\xi_{\mathrm{i}}(l, t)(i=1,2,3,4,5,6)$ - функции Лагранжа; пользуясь аргументацией вариационного исчисления, найдем вариацию функционала $I$ при оптимальных $\rho, x, u, T_{\text {п }}, T_{C}^{1}, T_{C}^{2}$ и получим сопряженную систему относительно $\xi_{i}, i=\overline{1,6}$ : 


$$
\left\{\begin{array}{l}
\frac{\partial \xi_{1}}{\partial t}=-\xi_{4} \frac{Q\left(T_{\mathrm{n}}, T_{C}^{1}, T_{C}^{2}\right)}{C_{\nu} \rho^{2}}-u \frac{\partial \xi_{1}(l, t)}{\partial l}-\frac{R T_{\mathrm{n}}}{\rho} \frac{\partial \xi_{3}(l, t)}{\partial l}-\frac{\xi_{3} R}{\rho} \frac{\partial T_{\mathrm{n}}(l, t)}{\partial l}, \\
\frac{\partial \xi_{2}}{\partial t}=\xi_{2} \frac{1}{\tau}-\xi_{4} \frac{q}{C_{v} \tau}-u \frac{\partial \xi_{2}(l, t)}{\partial l}-\xi_{2} \frac{\partial u(l, t)}{\partial l}, \\
\frac{\partial \xi_{3}}{\partial t}=\xi_{2} \frac{\partial x(l, t)}{\partial l}-\rho \frac{\partial \xi_{1}}{\partial l}-u \frac{\partial \xi_{3}}{\partial l}+(1-\gamma)\left[T_{\mathrm{n}} \frac{\partial \xi_{4}}{\partial l}+\xi_{4} \frac{\partial T_{\mathrm{n}}}{\partial l}\right]+\xi_{4} \frac{\partial T_{\mathrm{n}}}{\partial l}, \\
\frac{\partial \xi_{4}}{\partial t}=\xi_{3} \frac{R}{\rho} \frac{\partial \rho(l, t)}{\partial l}+(\gamma-2) \xi_{4} \frac{\partial u(l, t)}{\partial l}+\left[\frac{1}{C_{\nu} \rho} \frac{\partial Q\left(T_{\mathrm{n}}, T_{C}^{1}, T_{C}^{2}\right)}{\partial T_{\mathrm{n}}}+K_{1}\right] \xi_{4} \\
-\xi_{5}\left[K_{2}-\frac{\partial Q\left(T_{\mathrm{n}}, T_{C}^{1}\right)}{\partial T_{\mathrm{n}}}\right]+\left[-K_{2}+\frac{\partial Q\left(T_{\mathrm{n}}, T_{C}^{1}\right)}{\partial T_{\mathrm{n}}}\right], \\
\frac{\partial \xi_{5}}{\partial t}=\xi_{5}\left[K_{2}+\frac{\partial Q\left(T_{\mathrm{n}}, T_{C}^{1}, T_{C}^{2}\right)}{\partial T_{C}^{2}}\right]+2\left(T_{C}^{1}-T_{C}^{1^{*}}\right)-\omega \frac{\partial \xi_{5}(l, t)}{\partial l}-\xi_{4}\left[K_{1}+\frac{\partial Q\left(T_{\mathrm{n}}, T_{C}^{1}, T_{C}^{2}\right)}{\partial T_{C}^{1}}\right], \\
\frac{\partial \xi_{6}}{\partial t}+\omega \frac{\partial \xi_{6}}{\partial l}=\left[\frac{1}{C_{\nu} \rho} \frac{\partial Q\left(T_{\mathrm{n}}, T_{C}^{1}, T_{C}^{2}\right)}{\partial T_{C}^{2}}-K_{1}\right] \xi_{4}+\left[K_{2}+\frac{\partial Q\left(T_{\mathrm{n}}, T_{C}^{2}\right)}{\partial T_{C}^{2}}\right] \xi_{6} .
\end{array}\right.
$$

Начальные условия

$$
\xi_{i}(l, T)=0, i=\overline{1,5}
$$

Аналогично проведем преобразования вспомогательного функционала $I_{2}$ на границе области. В результате имеем:

$$
\begin{aligned}
& \left\{\begin{array}{l}
\frac{\mathrm{d} \lambda^{(1)}}{\mathrm{d} t}=-\xi_{1}(0, t) u(0, t)-\xi_{3}(0, t) \frac{R T_{\mathrm{\Pi}}(0, t)}{\rho(0, t)}, \\
\frac{\mathrm{d} \lambda^{(2)}}{\mathrm{d} t}=-\xi_{2}(0, t) u(0, t), \\
\frac{\mathrm{d} \lambda^{(3)}}{\mathrm{d} t}=-\xi_{1}(0, t) \rho(0, t)-\xi_{3}(0, t)+\xi_{4}(0, t)(1-\gamma) T_{\text {п }}(0, t), \\
\frac{\mathrm{d} \lambda^{(4)}}{\mathrm{d} t}=-\xi_{3}(0, t) R(0, t)-\xi_{4}(0, t) u(0, t), \\
\frac{\mathrm{d} \lambda^{(5)}}{\mathrm{d} t}=-\xi_{5}(L, t) \omega,
\end{array}\right. \\
& \lambda^{(1)}(T)=0, \quad \lambda^{(2)}(T)=0, \quad \lambda^{(3)}(T)=0, \quad \lambda^{(4)}(T)=0, \quad \lambda^{(5)}(T)=0, \\
& \xi_{1}(L, t) u(L, t)+\xi_{3}(L, t) \frac{R T_{\mathrm{\Pi}}(L, t)}{\rho(L, t)}=0, \\
& \xi_{2}(L, t) u(L, t)=0, \\
& \xi_{1}(L, t) \rho(L, t)+\xi_{3}(L, t) u(L, t)-(1-\gamma) \xi_{4}(L, t) T_{\mathrm{n}}(L, t)=0, \\
& \xi_{3}(L, t) R+\xi_{4}(L, t) u(L, t)=0 \text {, } \\
& \xi_{5}(0, t) \omega=0 \text {. }
\end{aligned}
$$


Из последних равенств следует, что $\xi_{i}(L, t)=0, i=1,2,5$,

$$
\begin{gathered}
\mu_{1} z_{1}(t)=0, \mu_{2} z_{2}(t)=0, \mu_{3} z_{3}(t)=0, \mu_{4} z_{4}(t)=0, \mu_{5} z_{5}(t)=0, \\
-\lambda^{(i)} b_{i}+\mu^{(i)}\left(v_{i \max }-2 v_{i}(t)+v_{i \max }\right)=0 \\
i=\overline{1,5} .
\end{gathered}
$$

Таким образом, мы получили систему (9) с начальными условиями (10) и граничными условиями (11)-(14).

Из (13) следует: если $z_{i} \equiv 0, i=\overline{1,5}$, то управления $v_{i}(t), i=\overline{1,5}$, принимают граничные значения $v_{i \min }$ или $v_{i \max }$.

Левые части конечно-разностных аналогов уравнений (14) представляют собой градиенты аппроксимированного функционала качества (6). Следовательно, для решения задачи может быть применен градиентный метод.

Метод решения задачи оптимального управления заключается в следующем:

1. Задаются начальные приближения управления $v_{i}^{0}(t), i=\overline{1,5}$.

2. Если $v_{i}^{n}(t)$ известны, из системы уравнений (1) и начальных (2) и граничных (3) условий находят $\rho^{n}=\rho(l, t), x^{n}(l, t), u^{n}(l, t), T_{I}^{n}(l, t), T_{C}^{n}(l, t)$, и из сопряженной задачи (9)-(14) находят $\xi_{i}^{n}(l, t), \lambda_{i}^{n}(t), i=\overline{1,5}$.

3. Далее полагаем $v_{i}^{n+1}=v_{i}^{n}-\tau L_{i}, n=0,1,2, \ldots, i=\overline{1,5}$.

4. Предельные значения управлений дают решение задачи оптимального управления.

Рассмотрим задачу оптимального управления расходом сырья $F$ в ректификационной колонне.

Для получения необходимых условий оптимальности (условий стационарности) используются методы классического вариационного исчисления. Управления предполагаются кусочнонепрерывными, а соответствующие им решения - непрерывными и кусочно-гладкими. Здесь рассматривается следующая модель процесса [1]:

$$
\begin{aligned}
& \frac{\mathrm{d}\left(H_{x} x\right)}{\mathrm{d} t}-\frac{\mathrm{d}(L x)}{\mathrm{d} l}=k\left(y-y^{*}(x)\right)+F(t) \Phi_{x}(l) x_{F}, \\
& \frac{\mathrm{d}\left(H_{y} y\right)}{\mathrm{d} t}-\frac{\mathrm{d}(V y)}{\mathrm{d} l}=k\left(y^{*}(x)-y\right), \quad 0<t<T, \quad 0<l<L,
\end{aligned}
$$

краевые условия:

при $l=0$

при $l=L$

$$
\begin{aligned}
& \frac{\mathrm{d}\left(H_{x_{k}} x_{k}\right)}{\mathrm{d} l}=L(0, t) x(0, t)-V(0, t) y(0, t)-W x_{k}(t), \\
& y(0, t)=a\left[y_{k}^{*}\left(x_{k}\right)-x_{k}(t)\right]+x_{k}(t) .
\end{aligned}
$$

$$
\begin{gathered}
\frac{\mathrm{d}\left(H_{x_{d}} x_{d}\right)}{\mathrm{d} l}=V_{d}(t) y_{d}(t)-\left(L_{d}(t)+D(t)\right) x_{d}(t), \\
V_{d} y_{d}(t)-V(L, t) y(L, t)=L_{d} x_{d}(t)-L(L, t) x(L, t), \\
y_{d}(t)=y(L, t)+E_{d}\left[y_{d}^{*}\left(x_{d}\right)-x(L, t)\right] ; \\
-101-
\end{gathered}
$$


начальные условия:

$$
\begin{aligned}
& x(l, 0)=x_{0}(l), y(l, 0)=y_{0}(l), 0<l<1, \\
& x_{d}(0)=x_{d 0}, x_{k}(0)=x_{k 0}, 0 \leq a, E_{d} \leq 1 .
\end{aligned}
$$

$F(t)$ - поток сырья в жидкой фазе, подводимый в колонну и являющийся управлением.

Здесь $x(l, t), y(l, t)$ - концентрации целевого продукта в жидкой и паровой фазах; $L(l, t)-$ поток жидкости; $V(l, H)$ - поток пара; $H_{x}, H_{y}$ - удерживающие способности в жидкости и паре; $x_{d}$ - концентрация целевого продукта в дефлегматоре; $x_{k}$ - концентрация целевого продукта в кубе. Возмущающее воздействие по концентрации целевого продукта в сырье $x_{F 2}($ рис. $2 a), y^{*}-$ равновесная концентрация целевого продукта в паровой фазе; $D, W$-отбор целевого продукта вверху и внизу колонны; $L_{d}$ - орошение.

Величины потоков при этом удовлетворяют условиям

$$
\begin{aligned}
& W(t)+D(t)=F(t), D(t)+L_{d}(t)=V(1, t), \\
& W(t)+V(0, t)=L(0, t), V_{d}(t)=V(1, t) .
\end{aligned}
$$

Предполагается, что удерживающие способности $H_{x}, H_{y}$ постоянны, $V$ не зависит от $l$.

Анализ условий (19) показывает, что только два из четырех потоков $(W, D, L, V)$ являются независимыми. Выбор тех или иных двух независимых потоков в качестве управлений определяет соответствующие задачи. Эти задачи рассматриваются как задачи оптимального управления в классе кусочно-непрерывных управлений с критерием качества.

$$
I=\int_{0}^{T} \int_{0}^{L}\left(y(l, t)-\theta^{*}(l, t)\right)^{2} \mathrm{~d} l \mathrm{~d} t,
$$

где $\theta^{*}$ - заданное значение концентрации целевого продукта.

Потоки $L, D$ фиксированы, а $V, V_{d}, L_{d}$ исключаются согласно (19). Управление $F$ выбирается в классе кусочно-непрерывных функций и принимает значение в промежутке

$$
F_{\min }<F(t)<F_{\max }
$$

Переходя к нормальной форме дифференциальных уравнений (15)-(17), получаем следующую задачу. Рассматривается процесс ректификации, описываемый уравнениями

$$
\begin{aligned}
& x_{t}^{\prime}=\frac{1}{H_{x}}\left[\left(L+L^{*}\right) \varsigma^{(1)}+\frac{\partial L^{*}}{\partial l} x+k\left(y-y^{*}\right)+F x_{F} \Phi_{x}\right] \equiv X, \quad x_{1}^{\prime}=\varsigma^{(1)}, \\
& y_{t}^{\prime}=\frac{1}{H_{y}}\left[-(D+L) \varsigma^{(2)}+k\left(y^{*}-y\right)\right] \equiv Y, \quad y_{1}^{\prime}=\varsigma^{(2)}
\end{aligned}
$$

при краевых условиях:

$$
\begin{array}{r}
x_{k t}^{\prime}=\frac{1}{H_{x_{k}}}\left[(L+F) x-V y-W x_{k}\right] \equiv X_{k}, \\
y=a\left[y_{k}^{*}-x_{k}\right]+x_{k}, \quad l=0, \quad 0<t<T, \\
-102-
\end{array}
$$




$$
\begin{gathered}
x_{d t}^{\prime}=\frac{1}{H_{x_{d}}}\left[(D+L) y_{d}-(L+D) x_{d}\right] \equiv X_{d}, \quad l+L, \quad 0<t<T, \\
(D+L)\left(y_{d}-y\right)-L\left(x_{d}-x\right)=0, \quad y_{d}-y-E_{d}\left(y_{d}^{*}-y\right)=0 .
\end{gathered}
$$

при начальных условиях (20) и ограничениях на управления

$$
\left(F-F_{\min }\right)\left(F_{\max } F\right)-u^{2}=0
$$

( $u$ - вспомогательное управление).

Задача состоит в том, чтобы во множестве кусочно-непрерывных функций $F$, удовлетворяющих условию (23), найти такую, что соответствующее ей решение задачи (21)-(23), (18) дает минимум интегралу (20). Применяя известную процедуру вариационного исчисления, получаем необходимое условие оптимальности.

$$
\begin{aligned}
& \xi_{t}^{\prime}-\frac{L+L^{*}}{H_{x}} \xi_{l}^{\prime}=k\left(y^{*}\right)^{\prime}\left(\frac{\xi}{H_{x}}-\frac{\eta}{H_{y}}\right), \\
& \eta_{t}^{\prime}+\frac{V}{H} \eta_{l}^{\prime}=k\left(\frac{\xi}{H_{x}}-\frac{\eta}{H_{y}}\right)
\end{aligned}
$$

при $l=0,0<t<T$,

$$
\begin{aligned}
& \frac{\mathrm{d} \lambda_{k}^{(1)}}{\mathrm{d} t}=\frac{\lambda_{k}^{(1)}}{H_{x_{k}}}(F-D)+\lambda_{k}^{(2)}\left(a-1-a\left(y_{k}^{*}\right)\right), \quad \lambda_{k}^{(1)}(T)=0, \\
& \lambda_{k}^{(1)}(L+F)-\xi\left(L+L^{*}\right)=0, \quad V\left(\frac{\eta}{H_{y}}-\frac{\lambda_{k}^{(1)}}{H_{x_{k}}}\right)-\lambda_{k}^{(2)}=0 ;
\end{aligned}
$$

при $t=T, 0<l<L \xi=0, \eta=0$; при $l=L, 0<t<T$

$$
\begin{aligned}
& \frac{\mathrm{d} \lambda_{d}^{(1)}}{\mathrm{d} t}=\frac{L+D}{H_{x_{d}}} \lambda_{d}^{(1)}-L \lambda_{d}^{(2)}, \quad \lambda_{d}^{(1)}(T)=0, \quad-\frac{V}{H_{x_{d}}} \lambda_{d}^{(1)}+\lambda_{d}^{(2)} V+\lambda_{d}^{(3)}=0, \\
& \left(\lambda_{d}^{(2)}-\frac{\xi}{H_{x_{d}}}\right) L-\frac{\xi}{H_{x_{d}}} L^{*}-\lambda_{d}^{(3)} E_{d}\left(y_{d}^{*}\right)=0, \quad\left(\frac{\eta}{H_{y}}-\lambda_{d}^{(2)}\right) V-\lambda_{d}^{(3)}\left(1-E_{d}\right)=0 ;
\end{aligned}
$$

при $0<t<T$

$$
H=\int_{0}^{1}-\frac{\xi}{H_{x}}\left(\frac{\partial^{2} L^{*}}{\partial l \partial F} x+\Phi_{x} x_{F}-\frac{\partial L^{*}}{\partial F} \xi^{(1)}\right) \mathrm{d} l-\frac{\lambda_{k}^{(1)}}{H_{x_{k}}}\left(x_{k}-x\right)+\gamma\left(F_{\min }+F_{\max }-2 F\right)=0
$$

где $\xi, \eta, \lambda_{d}^{(1)}, \lambda_{d}^{(2)}, \lambda_{d}^{(3)}, \lambda_{k}^{(1)}, \lambda_{k}^{(2)}-$ множители Лагранжа.

Алгоритм решения задачи оптимального управления содержит следующие этапы:

1) задается начальное приближение управляющей функции $F^{\circ}(t)$;

2) решается система уравнений (15)-(18) и (24)-(26);

3) далее полагаем $F^{(n+1)}=F^{(n)}-\alpha H$;

4) предельные значения $F^{(n+1)}$ при $n \rightarrow \infty$ дают оптимальные управления. 


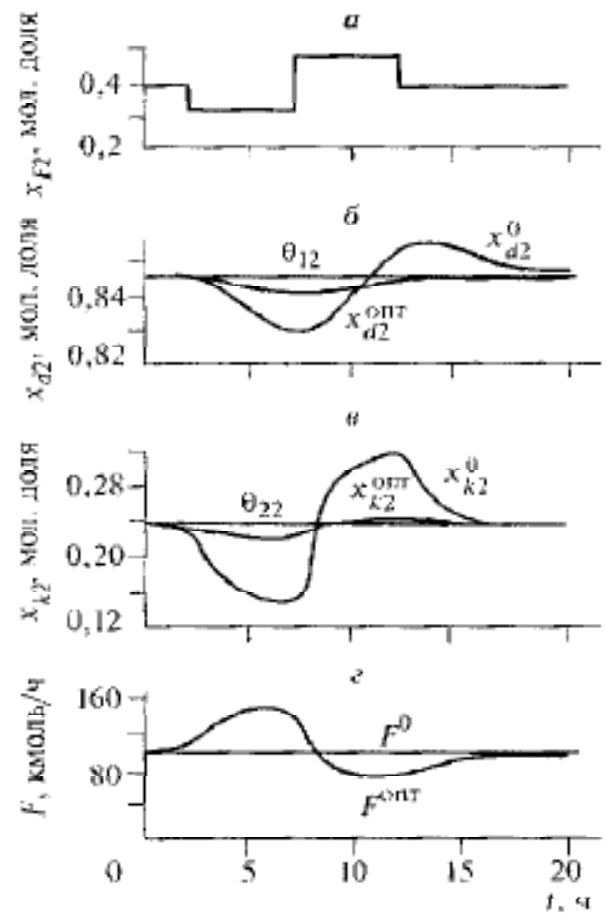

Рис. 2. Графики изменения концентрации бутана в сырье (a), дефлегматоре (б) и кубе (в) при управлении потоком сырья (2)

На рис. 2 приведены результаты расчетов по оптимальному управлению для промышленной колонны К-34 [1] установки сернокислотного алкилирования изобутана бутиленами (разделяемая многокомпонентная смесь сведена к бинарной). Основные параметры: $D=27,06$ кмоль $/$ ч, $W=76,59$ кмоль $/$, $L_{d}=45,21$ кмоль $/$ ч, $H x_{d}=50$ кмоль, $\mathrm{H} x_{k}=30$ кмоль.

Исследование выполнено при финансовой поддержке Российского фонда фундаментальных исследований, Правительства Красноярского края, Красноярского краевого фонда поддержки научной и научно-технической деятельности в рамках научного проекта № 16-41-242156р_oфu_м.

\section{Список литературы}

[1] Демиденко Н.Д., Кулагина Л.В. Особенности сжигания топлива в технологических установках. Химическая техника, 2006, 7, 35. [Demidenko N.D., Kulagina L.V. Features of fuel combustion in process plants. Chemical engineering, 2006, 7, 35 (in Russian)]

[2] Демиденко Н.Д., Кулагина Л.В. Оптимальное управление режимами работы технологических печей в нефтеперерабатывающей промышленности, Фундаментальные исследования, 2005, 2, 43-44. [Demidenko N.D., Kulagina L.V. Optimal control of operating modes of process furnaces in the refining industry, Basic Research, 2005, 2, 43-44. (in Russian)]

[3] Демиденко Н.Д., Кулагина Л.В. Методы и средства оптимального управления теплотехнологическими процессами в трубчатых печах. Химическое и нефтегазовое машино- 
строение, 2006, 3, 8-9. [Demidenko N.D., Kulagina L.V. Methods and tools for of optimal control thermal technological processes in tube furnaces. Chemical and Petroleum Engineering, 2006, 3, 8-9 (in Russian)]

[4] Demidenko N.D., Kulagina L.V. Optimal Control of Thermalengineering Processes in Tube Furnaces. Chemical and Petroleum engineering, 2006, 42(3/4), 128-130.

[5] Демиденко Н.Д., Кулагина Л.В. Численное исследование систем с распределенными параметрами. Вестник КрасГАУ, 2007, 2, 103-112. [Demidenko N.D., Kulagina L.V. Numerical study of systems with distributed parameters. KrasGAU Vestnik, 2007, 2, 103-112 (in Russian)]

[6] Демиденко Н.Д., Кулагин В.А., Шокин Ю.И., Ли Ф.-Ч. Тепломассообмен и суперкавитация. Новосибирск: Наука, 2015, 436 с. [Demidenko N.D., Kulagin V.A., Shokin Y.I., Lee F.-CH. Heat and Mass Transfer and Supercavitation. Novosibirsk, Nauka, 2015. 436 p. (in Russian)]

[7] Демиденко Н.Д., Кулагина Л.В. Моделирование и оптимизация технических систем с распределенными параметрами. Красноярск: ИПЦ КГТУ, 2006. 210 с. [Demidenko N.D., Kulagina L.V. Simulation and Optimization of Engineering Systems with Distributed Parameters. Krasnoyarsk, Publishing Center KSTU, 2006. 210 p. (in Russian)]

[8] Demidenko N.D., Kulagina L.V. Stationary and dynamic processes in process furnaces, J. Sib. Fed. Univ. Eng. technol., 2016, 9(1), 6-14. DOI: 10.17516/1999-494X-2016-9-1-6-14. 